\title{
Adiponectin correlates with body mass index and to a lesser extent with left ventricular mass in dialysis patients
}

\author{
Afrim Poniku, ${ }^{1,2}$, Gani Bajraktari, ${ }^{1,2}$, Shpend Elezi², \\ Pranvera Ibrahimi ${ }^{1,3}$, Michael Y. Henein ${ }^{3}$ \\ ${ }^{1}$ Clinic of Cardiology, University Clinical Centre of Kosova, Prishtina, Kosovo \\ ${ }^{2}$ Medical Faculty, University of Prishtina, Prishtina, Kosovo \\ ${ }^{3}$ Department of Public Health and Clinical Medicine, Umeå University and Heart Centre, Umeå, Sweden
}

\begin{abstract}
Background: Adiponectin is a serum protein produced by adipose tissue which exerts anti-inflammatory, anti-diabetic and anti-atherosclerotic properties, hence is considered a cardio-protective marker. With the current uncertain role of adiponectin in dialysis patients to the aim of this study was to investigate its relationship with left ventricular (LV) structure and function in these patients.

Methods: This study included 89 (age $56 \pm 13$ years, $43 \%$ male) patients treated with regular dialysis for $>6$ months, and 55 control subjects with normal renal function. A complete two-dimensional, $M$-mode and tissue-Doppler echocardiographic study, and biochemical blood analyses, adiponectin and anthropometric parameters were obtainedon the same day.

Results: Dialysis patients had lower body mass index (BMI) and lower body surface area (BSA) $(p<0.001$ for both), lower waist/hips ratio $(p=0.005)$, higher LV mass index (LVMI, $p<0.001)$, higher adiponectin level $(p<0.001)$ and LV end-systolic volume $(p=0.003)$, lower LV ejection fraction $(p=0.006)$, longer isovolumic relaxation time $(p<0.001)$, lower mean LV strain $(p=0.002)$, larger left atrium volume $(p=0.022)$ and lower left atrium emptying fraction $(p=0.026)$, compared to controls. In dialysis patients, adiponectin correlated with waist circumference $(r=-0.427, p<0.001), B M I$ $(r=-0.403, p<0.001)$ and BSA $(r=-0.480, p<0.001)$, and to a lesser extent with $L V M I(r=0.296$, $p=0.005)$, waist $/$ hips ratio $(r=-0.222, p=0.037)$ and total cholesterol $(r=-0.292, p=0.013)$. But in controls, it correlated only modestly with age $(r=0.304, p=0.024)$, hemoglobin $(r=0.371$, $p=0.005)$, high density lipoprotein cholesterol $(r=0.315, p=0.019)$ and LVMI $(r=0.277, p=0.043)$. Conclusions: It seems that in dialysis patients, adiponectin modest correlation with anthropometric measurements suggests an ongoing catabolic process rather than a change in ventricular function. (Cardiol J 2018; 25, 4: 501-511)
\end{abstract}

Key words: adiponectin, dialysis, left ventricular mass, body mass index

\section{Introduction}

Adiponectin, an adipocyte-derived hormone, is a serum protein produced by the adipose tissue $[1,2]$, and exerts anti-inflammatory, anti-diabetic and anti-atherosclerotic properties [3-6], and hence is considered a cardio-protective marker. Several previous studies suggested a possible influence of adiponectin in many clinical conditions, such as obesity, insulin resistance, hypertension, dyslipidemia and atherosclerotic heart disease [7-11]. Hypoadiponectinemia has been identified as independent risk factor for cardiovascular $(\mathrm{CV})$ disease $[12,13]$. Adiponectin prevents progression of left

Address for correspondence: Gani Bajraktari, MD, FESC, FACC, Public Health and Clinical Medicine, Umeå University, Umeå, Sweden, tel: +46907850000, +377 45 800808, e-mail: gani.bajraktari@umu.se 
ventricular (LV) hypertrophy (LVH) $[4,14]$, which correlates with CV complications both in hypertensive $[15,16]$ and chronic kidney disease (CKD) $[17,18]$. While, it was found to normally correlate with blood pressure values, such a relationship in hypertensives remains controversial [11, 19-22]. On the other hand, adiponectin levels have been found to correlate with LV mass index (LVMI) $[4,23]$, and with impaired renal function [24-26], in patients with renal failure and those treated with dialysis, although modestly [24,27]. The aim in this study therefore, was to investigate the relationship between adiponectin and LV structure and function measurements in dialysis patients, as an attempt to get more insight into its protective $\mathrm{CV}$ role in these patients.

\section{Methods}

\section{Patients}

Eighty-nine consecutive patients treated with regular dialysis at the Dialysis Unit of the University Clinical Centre of Kosovo were included in this study (age $56 \pm 13$ [18-80] years, $43 \%$ male) and 55 subjects, who had normal renal function, and served as a control group which was recruited between May 2013 and April 2016. All subjects gave informed consent to participate in the study, which was approved by the Ethics Committee of Medical Faculty, University of Prishtina. All patients included had been receiving chronic standardized dialysis $500-750 \mathrm{~mL} / \mathrm{min}$ dialysate flow, $250-300 \mathrm{~mL} / \mathrm{min}$ blood flow, over 3-4 h session, 3 times per week, for at least 6 months prior to recruitment in the study. The dialysis potassium level was $2.0 \mathrm{mEq} / \mathrm{L}$ and calcium level was $1.75 \mathrm{mEq} / \mathrm{L}$. Patients with active malignancy, decompensated heart failure, hepatic or pulmonary disease, pregnant women and those with failed transplant were excluded from the study. Blood pressure was recorded with a brachial sphygmomanometer after subjects had rested in the supine position for at least $10 \mathrm{~min}$.

\section{Clinical data}

For all participants, demographic details, physical examination and anthropometric measurements were taken. Hypertension was defined as systolic blood pressure $\geq 140 \mathrm{mmHg}$, and/or diastolic blood pressure $\geq 90 \mathrm{~mm} \mathrm{Hg}$, or when patients were using antihypertensive therapy. Diabetes was defined as fasting plasma glucose $\geq 126 \mathrm{mg} / \mathrm{dL}$ $(>7 \mathrm{mmol} / \mathrm{L}$ ) or the use of hypoglycemic medications. Body mass index (BMI) was measured after the dialysis session and was calculated by dividing dry weight by body height $\left(\mathrm{kg} / \mathrm{m}^{2}\right)$. Twelve lead electrocardiogram was also recorded in all patients and QRS duration was measured.

\section{Blood analysis}

Blood urea nitrogen, creatinine, albumin, total protein, total cholesterol, triglyceride, calcium, phosphate, iron, hematocrit and hemoglobin were measured using standard methods. High density lipoprotein cholesterol (HDL-L) was measured by homogeneous enzymatic colorimetric assay (COBAS INTEGRA $^{\circledast} 400$ plus, Roche), parathyroid hormone by immunoassay (COBAS e 411 analyzerRoche) and C-reactive protein (CRP) by particle enhanced turbidimetric assay (COBAS INTEGRA ${ }^{\circledast}$ 400 plus, Roche). All samples for a given assay were tested simultaneously, in duplicate and in appropriate dilutions, according to conventional protocols.

\section{Adiponectin measurement}

Venous blood samples were withdrawn from each subject after $\geq 8 \mathrm{~h}$ of fasting. The samples were stored at $-80^{\circ} \mathrm{C}$ until analyzed. Serum adiponectin concentration was measured by ELISA Microplate Reader Gea Linear. Intra- and interassay coefficient of variation was below $3.0 \%$ and $5.1 \%$, respectively [28].

\section{Cardiac structure and function:}

Left ventricular structure measurements. Echocardiographic examination in all patients and in controls was performed on the same day of dialysis using a Philips iE33 system with a multifrequency transducer and harmonic imaging as appropriate. Images were obtained with the patient in the left lateral decubitus position and during quiet expiration. $\mathrm{LV}$ volumes and ejection fraction $(\mathrm{EF})$ were calculated from the apical 2- and 4-chamber views using the modified Simpson method. Left ventricular mass (LVM) was estimated using the anatomically validated formula of Devereux et al. $[29,30]$ and was indexed to height ${ }^{2.7}$ (LVMI) [31]: LVM $[\mathrm{g}]=0.8 \times(1.04 \times(\mathrm{LVEDD}+\mathrm{PWTD}+$ + IVSTD $\left.)^{3}-(\text { LVEDD })^{3}\right)+0.6[30]$. LVH was defined as a LVMI of $47 \mathrm{~g} / \mathrm{m}^{2.7}$ for women and $53 \mathrm{~g} / \mathrm{m}^{2.7}$ for men [32]. LVM normalized for body surface area (LVM/BSA) was also calculated as $\mathrm{g} / \mathrm{m}^{2}$.

Left ventricular function measurements. From the spectral wave Doppler LV filling, peak E wave velocity, peak A wave velocity, the ratio between peak $\mathrm{E}$ and $\mathrm{A}$ velocities (E/A ratio), and $\mathrm{E}$ wave deceleration time were measured. From tissue-Doppler imaging recordings, peak systolic (s'), 
and early diastolic (e') and late diastolic (a') mitral annular velocities were also measured. The ratio of trans-mitral to myocardial early diastolic peak velocity (E/e') was calculated, after averaging septal and lateral e'velocities [33, 34], to reflect filling pressures. Mitral annular plane systolic excursion (MAPSE) was measured by placing the M-mode cursor at the lateral and septal angles.

Total LV filling time (FT) was measured from the onset of the $\mathrm{E}$ wave to the end of the $\mathrm{A}$ wave and ejection time (ET) from the onset to the end of the aortic Doppler flow velocity. Total isovolumic time (t-IVT) was calculated as 60 - (total ET + total FT) and was expressed in $\mathrm{s} / \mathrm{min}$ [35]. Tei index was calculated as the ratio between t-IVT and ET [36]. $\mathrm{LV}$ isovolumic relaxation time (IVRT) was measured as the time interval between LV end-ejection (from the pulsed-wave Doppler recording of the outflow tract velocity) and the onset of transmitral E-wave velocity.

The diameter of the LV outflow tract (LVOT) was measured from the parasternal long axis view as the distance between the bases of the aortic valve cusp during systole. The LVOT area was then calculated using the formula: LVOT area $=$ $=\left[(\text { LVOT diameter average } / 2)^{2}\right] \times 3.14$.

The average velocity time integral (VTI) was measured using the pulsed wave Doppler samples obtained at the center of the LVOT from the apical view. The stroke volume was calculated as the product of the LVOT area and the VTI of the LVOT blood flow.

Left atrial (LA) measurements. LA diameter was measured from aortic root recordings with the M-mode cursor positioned at the level of the aortic valve leaflets. LA volumes were measured using area-length method from the apical four chamber views, according to the guidelines of the American Society of Echocardiography and European Association of Echocardiography [22, 37]. Left atrial maximal volume (LAV max) was measured at the end of LV systole, just before the opening of the mitral valve, LA minimal volume (LAV min) was measured at end diastole, immediately after mitral valve closure. LA total emptying fraction was calculated using the formula [38]: LA total emptying fraction $=\mathrm{LAV} \max -\mathrm{LAV} \min / \mathrm{LAV} \max \times 100$.

\section{Statistical analysis}

Values are expressed as means \pm standard deviation. Differences between the two groups were analyzed using the unpaired Student $t$ test following the analysis of variance. $\mathrm{P}$ values $<0.05$ were considered statistically significant. The $\chi^{2}$ test was used to compare the categorical variables. Pearson correlations were performed to identify the simple correlations between adiponectin and the other variables. Subjects were stratified into four groups based on the dialysis treatment and on the presence of $\mathrm{LVH}$ on the echocardiographic examination: Group 1 (dialysis patients with LVH), Group 2 (dialysis patients without LVH), Group 3 (non-dialysis patients with $\mathrm{LVH}$ ) and Group 4 (non-dialysis patients without LVH). A one-way ANOVA with Bonferroni correction was performed to compare continuous variables. All analyses were performed using SPSS 22 for windows.

\section{Results}

\section{Clinical data of dialysis patients versus controls (Table 1)}

The baseline demographic, anthropometric and clinical data of dialysis patients and controls are shown in Table 1 . The age, gender and CV risk factors (smoking, diabetes, arterial hypertension and cholesterol level) were not different between groups. The dialysis patients had lower BMI and lower BSA ( $<0.001$ for both), smaller waist measurements $(\mathrm{p}<0.001)$, smaller interhip distance $(\mathrm{p}=0.002)$, lower waist/hip ratio ( $\mathrm{p}$ $=0.005)$, higher SBP and DBP $(\mathrm{p}=0.002$ and $\mathrm{p}=0.018$, respectively), higher baseline heart rate, higher CRP level, lower red blood cell (RBC), lower hemoglobin, lower albumin level, lower hematocrit and higher adiponectin level $(\mathrm{p}<0.001$, for all), lower blood iron level $(\mathrm{p}=$ $=0.028)$, and higher fasting glucose $(\mathrm{p}=0.005)$ compared to controls. Less dialysis patients were treated with beta-blockers ( $p<0.001)$, and more treated with angiotensin converting enzyme inhibitors $(\mathrm{p}=0.003)$ and calcium antagonists $(\mathrm{p}<0.001)$, compared to controls.

\section{Cardiac function of dialysis patients versus controls (Table 2)}

Dialysis patients had higher LVMI $(\mathrm{p}<0.001)$, thicker interventricular septum $(\mathrm{p}=0.006)$, larger LV end-systolic volume $(\mathrm{p}=0.003)$, lower LVEF $(\mathrm{p}=0.006)$, shorter LVET $(\mathrm{p}<0.001)$, longer IVRT $(\mathrm{p}<0.001)$, lower Tei index $(\mathrm{p}=0.003)$, lower septal MAPSE $(\mathrm{p}=0.036)$, higher A wave $(p=0.009)$, lower septal e' $(p=0.017)$, larger LA volume $(\mathrm{p}=0.022)$ and lower LA emptying fraction $(\mathrm{p}=0.026)$, compared to controls. 
Table 1. Clinical and biochemical data in dialysis patients versus controls.

\begin{tabular}{|c|c|c|c|}
\hline Variable & Controls $(n=55)$ & Dialysis patients $(n=89)$ & $\mathbf{P}$ \\
\hline Age [years] & $55 \pm 13$ & $56 \pm 13$ & 0.839 \\
\hline Gender [male, \%] & 56 & 43 & 0.111 \\
\hline Smoking [\%] & 31 & 20 & 0.146 \\
\hline Diabetes [\%] & 11 & 18 & 0.252 \\
\hline Hypertension [\%] & 40 & 56 & 0.059 \\
\hline $\mathrm{SBP}[\mathrm{mm} \mathrm{Hg}]$ & $141 \pm 20$ & $153 \pm 24$ & 0.002 \\
\hline $\mathrm{DBP}[\mathrm{mm} \mathrm{Hg}]$ & $87 \pm 10$ & $82 \pm 13$ & 0.018 \\
\hline Beta-blockers [\%] & 29 & 5.6 & $<0.001$ \\
\hline ACE-inhibitor [\%] & 24 & 47 & 0.003 \\
\hline Rocaltrol [\%] & 0 & 95 & $<0.001$ \\
\hline Calcium carbonate [\%] & 4 & 98 & $<0.001$ \\
\hline Aspirin [\%] & 42 & 9 & $<0.001$ \\
\hline Ca-channel blockers [\%] & 2 & 29 & $<0.001$ \\
\hline Diuretic [\%] & 20 & 10 & 0.096 \\
\hline Heart rate $[\mathrm{bpm}]$ & $73 \pm 10$ & $80 \pm 13$ & $<0.001$ \\
\hline Waist $[\mathrm{cm}]$ & $96 \pm 11$ & $88 \pm 12$ & $<0.001$ \\
\hline Hips [cm] & $105 \pm 11$ & $99 \pm 12$ & 0.002 \\
\hline Waist/hip ratio & $0.92 \pm 0.06$ & $0.89 \pm 0.05$ & 0.005 \\
\hline Weight $[\mathrm{kg}]$ & $80 \pm 13$ & $67 \pm 14$ & $<0.001$ \\
\hline Body mass index $\left[\mathrm{kg} / \mathrm{m}^{2}\right]$ & $27 \pm 3.4$ & $24 \pm 4.4$ & $<0.001$ \\
\hline Body surface area $\left[\mathrm{m}^{2}\right]$ & $1.1 \pm 0.2$ & $0.9 \pm 0.2$ & $<0.001$ \\
\hline Adiponectin $[\mu \mathrm{g} / \mathrm{mL}]$ & $5.9 \pm 3$ & $15 \pm 9$ & $<0.001$ \\
\hline C-reactive protein [mg/L] & $3.4 \pm 3$ & $11 \pm 20$ & $<0.001$ \\
\hline HDL-cholesterol [mmol/L] & $1.0 \pm 0.5$ & $0.9 \pm 0.3$ & 0.097 \\
\hline Total cholesterol $[\mathrm{mmol} / \mathrm{L}]$ & $4.8 \pm 0.8$ & $4.5 \pm 0.9$ & 0.108 \\
\hline Triglyceride $[\mathrm{mmol} / \mathrm{L}]$ & $1.6 \pm 0.5$ & $1.8 \pm 0.8$ & 0.030 \\
\hline Creatinine $[\mathrm{umol} / \mathrm{L}]$ & $80 \pm 16$ & $711 \pm 160$ & $<0.001$ \\
\hline Blood urea nitrogen [mmol/L] & $5.4 \pm 1.4$ & $27 \pm 5.3$ & $<0.001$ \\
\hline Parathormone $[\mathrm{pg} / \mathrm{mL}]$ & $39 \pm 26$ & $126 \pm 114$ & 0.012 \\
\hline Phosphorus [mg/dL] & $1.0 \pm 0.07$ & $1.6 \pm 0.5$ & $<0.001$ \\
\hline Total calcium $[\mathrm{mmol} / \mathrm{L}]$ & $2.2 \pm 0.15$ & $2.1 \pm 0.3$ & 0.428 \\
\hline Iron $[\mu \mathrm{mol} / \mathrm{L}]$ & $19 \pm 4.9$ & $16 \pm 8.3$ & 0.028 \\
\hline Fasting glucose $[\mathrm{mmol} / \mathrm{L}]$ & $5.6 \pm 1.5$ & $6.9 \pm 3.9$ & 0.005 \\
\hline Total protein $[\mathrm{g} / \mathrm{L}]$ & $66 \pm 2.6$ & $65 \pm 5.4$ & 0.438 \\
\hline Albumin $[\mathrm{g} / \mathrm{L}]$ & $40 \pm 4$ & $37 \pm 4$ & $<0.001$ \\
\hline Aspartate aminotransferase [U/L] & $18 \pm 6$ & $20 \pm 16$ & 0.291 \\
\hline Alanine aminotransferase $[\mathrm{U} / \mathrm{L}]$ & $20 \pm 8$ & $23 \pm 21$ & 0.148 \\
\hline Red blood cells $\times 10^{6} / \mathrm{L}$ & $4.5 \pm 0.6$ & $3.5 \pm 0.9$ & $<0.001$ \\
\hline White blood cells $\times 10^{3} / \mathrm{L}$ & $7.7 \pm 1.3$ & $7.5 \pm 3.2$ & 0.594 \\
\hline Hemoglobin $[\mathrm{g} / \mathrm{dL}]$ & $14 \pm 1.3$ & $10 \pm 1.6$ & $<0.001$ \\
\hline Hematocrit [\%] & $39 \pm 6.1$ & $33 \pm 5.5$ & $<0.001$ \\
\hline
\end{tabular}

SBP — systolic blood pressure; DBP — diastolic blood pressure; ACE — angiotensin converting enzyme; HDL — high-density lipoprotein 
Table 2. Echocardiographic data in controls group vs. dialysis patients.

\begin{tabular}{|c|c|c|c|}
\hline Variable & Controls $(n=55)$ & Dialysis patients $(n=89$ ) & $\mathbf{P}$ \\
\hline \multicolumn{4}{|l|}{ LV dimension and mass } \\
\hline LV mass [g] & $214 \pm 75$ & $230 \pm 66$ & 0.201 \\
\hline LV mass index $\left[\mathrm{g} / \mathrm{m}^{2.7}\right]$ & $47 \pm 13$ & $68 \pm 29$ & $<0.001$ \\
\hline Inter ventricular septum [cm] & $1.2 \pm 0.16$ & $1.25 \pm 0.2$ & 0.006 \\
\hline LV posterior wall [cm] & $1.0 \pm 0.1$ & $1.1 \pm 0.2$ & 0.095 \\
\hline End diastolic volume [mL] & $113 \pm 22$ & $119 \pm 35$ & 0.212 \\
\hline End systolic volume [mL] & $39 \pm 14$ & $48 \pm 23$ & 0.003 \\
\hline \multicolumn{4}{|l|}{ LV systolic function } \\
\hline LV ejection fraction [\%] & $64 \pm 7.7$ & $60 \pm 10$ & 0.006 \\
\hline LV shortening fraction [\%] & $34 \pm 5.1$ & $32 \pm 6.5$ & 0.071 \\
\hline Stroke volume [mL] & $72 \pm 19$ & $71 \pm 19$ & 0.809 \\
\hline Ejection time [ms] & $315 \pm 42$ & $279 \pm 42$ & $<0.001$ \\
\hline Tei index & $0.37 \pm 0.2$ & $0.36 \pm 0.3$ & 0.003 \\
\hline Lateral s' $[\mathrm{cm} / \mathrm{s}]$ & $7.1 \pm 1.9$ & $6.8 \pm 1.8$ & 0.468 \\
\hline Septal s' $[\mathrm{cm} / \mathrm{s}]$ & $6.1 \pm 1.5$ & $5.7 \pm 1.5$ & 0.142 \\
\hline Septal MAPSE [cm] & $1.4 \pm 0.3$ & $1.3 \pm 0.3$ & 0.036 \\
\hline Lateral MAPSE [cm] & $1.6 \pm 0.3$ & $1.5 \pm 0.3$ & 0.077 \\
\hline \multicolumn{4}{|l|}{ LV diastolic function } \\
\hline IVRT [ms] & $101 \pm 28$ & $127 \pm 30$ & $<0.001$ \\
\hline E wave $[\mathrm{cm} / \mathrm{s}]$ & $57 \pm 14$ & $56 \pm 18$ & 0.692 \\
\hline A wave $[\mathrm{cm} / \mathrm{s}]$ & $64 \pm 16$ & $72 \pm 18$ & 0.009 \\
\hline $\mathrm{E} / \mathrm{A}$ ratio & $0.9 \pm 0.6$ & $0.9 \pm 0.7$ & 0.783 \\
\hline EDT [ms] & $174 \pm 40$ & $178 \pm 50$ & 0.545 \\
\hline Lateral e' $[\mathrm{cm} / \mathrm{s}]$ & $8.1 \pm 2.9$ & $7.8 \pm 3.3$ & 0.627 \\
\hline Lateral $\mathrm{a}^{\prime}[\mathrm{cm} / \mathrm{s}]$ & $9.2 \pm 2.4$ & $9.3 \pm 2.9$ & 0.730 \\
\hline E/e' ratio & $8.3 \pm 2.3$ & $9.2 \pm 3.9$ & 0.092 \\
\hline Septal e' $[\mathrm{cm} / \mathrm{s}]$ & $6.5 \pm 2.0$ & $5.7 \pm 2.0$ & 0.017 \\
\hline Septal a' $[\mathrm{cm} / \mathrm{s}]$ & $7.9 \pm 1.5$ & $7.9 \pm 2.5$ & 0.921 \\
\hline \multicolumn{4}{|l|}{ LA dimension and function } \\
\hline LA diameter $[\mathrm{cm}]$ & $3.7 \pm 0.3$ & $3.9 \pm 0.5$ & 0.090 \\
\hline Maximal LA volume [mL] & $52 \pm 16$ & $56 \pm 22$ & 0.213 \\
\hline Minimal LA volume [mL] & $19 \pm 8$ & $23 \pm 14$ & 0.022 \\
\hline LA emptying fraction [\%] & $65 \pm 8$ & $60 \pm 11$ & 0.026 \\
\hline \multicolumn{4}{|l|}{ Aortic measurements } \\
\hline Aorta $[\mathrm{cm}]$ & $3.4 \pm 0.3$ & $3.4 \pm 0.4$ & 0.543 \\
\hline Ascending aorta $[\mathrm{cm}]$ & $3.5 \pm 0.3$ & $3.6 \pm 0.4$ & 0.125 \\
\hline
\end{tabular}

LV - left ventricular; LA — left atrium; MAPSE — mitral annular plane systolic excursion; IVRT — isovolumic relaxation time; RV — right ventricular; EDT - E wave deceleration time; A — atrial velocity; a' - late diastolic myocardial velocity; E - early mitral inflow velocity; $\mathrm{e}^{\prime}$ - early diastolic myocardial velocity; $\mathrm{s}^{\prime}$ - systolic myocardial velocity

Dialysis patients with $\mathrm{LVH}$ versus without LVH (Tables 3, 4)

In addition to the increased LVM and LVMI, the dialysis patients with LVH had lower weight, lower BSA, higher blood iron level, larger LV end diastolic volume, higher LVEF and LV stroke volume ( $\mathrm{p}<0.05$, for all) compared to dialysis patients without LVH. All the other clinical, biochemical and echocardiographic indices were not different between the two groups. 
Table 3. Clinical, anthropometrical and biochemical data in study subjects.

\begin{tabular}{|c|c|c|c|c|c|}
\hline Variable & $\begin{array}{l}\text { Group } 1 \\
(n=65)\end{array}$ & $\begin{array}{l}\text { Group } 2 \\
(n=24)\end{array}$ & $\begin{array}{l}\text { Group } 3 \\
(n=25)\end{array}$ & $\begin{array}{l}\text { Group } 4 \\
(n=30)\end{array}$ & $\mathbf{P}$ \\
\hline Age [years] & $56 \pm 13$ & $54 \pm 13$ & $59 \pm 13$ & $52 \pm 13$ & 0.186 \\
\hline $\mathrm{SBP}[\mathrm{mmHg}]$ & $155 \pm 25$ & $148 \pm 21$ & $146 \pm 21$ & $136 \pm 18^{3}$ & 0.004 \\
\hline $\mathrm{DBP}[\mathrm{mmHg}]$ & $83 \pm 14$ & $81 \pm 11$ & $88 \pm 10$ & $86 \pm 9$ & 0.098 \\
\hline Heart rate $[\mathrm{bpm}]$ & $80 \pm 13$ & $83 \pm 12$ & $78 \pm 11$ & $76 \pm 9$ & $<0.001$ \\
\hline Waist $[\mathrm{cm}]$ & $87 \pm 13$ & $92 \pm 10$ & $95 \pm 12^{2}$ & $97 \pm 11^{\mathrm{c}}$ & $<0.001$ \\
\hline Hips $[\mathrm{cm}]$ & $97 \pm 12$ & $103 \pm 11$ & $102 \pm 12$ & $107 \pm 9^{c}$ & $<0.001$ \\
\hline Waist/hips ratio & $0.89 \pm 0.06$ & $0.89 \pm 0.06$ & $0.93 \pm 0.06^{2}$ & $0.91 \pm 0.07$ & 0.030 \\
\hline Weight [kg] & $64 \pm 13$ & $74 \pm 13^{1}$ & $73 \pm 11^{2}$ & $85 \pm 12^{c, 5,6}$ & $<0.001$ \\
\hline Body mass index $\left[\mathrm{kg} / \mathrm{m}^{2}\right]$ & $24 \pm 5$ & $25 \pm 4$ & $27 \pm 3^{2}$ & $28 \pm 3^{c}$ & $<0.001$ \\
\hline Body surface area $\left[\mathrm{m}^{2}\right]$ & $0.9 \pm 0.2$ & $1.0 \pm 0.2^{1}$ & $1.0 \pm 0.18^{2}$ & $1.1 \pm 0.18^{\mathrm{c}, 5,6}$ & $<0.001$ \\
\hline Adiponectin $[\mu \mathrm{g} / \mathrm{mL}]$ & $16 \pm 8.6$ & $13 \pm 8.9$ & $6.8 \pm 2.5^{\mathrm{b}, 4}$ & $5.1 \pm 2.8^{\mathrm{c}, \mathrm{e}}$ & $<0.001$ \\
\hline C-reactive protein [mg/L] & $12 \pm 9$ & $9 \pm 4$ & $4 \pm 3$ & $3 \pm 2$ & 0.037 \\
\hline HDL-cholesterol [mmol/L] & $0.9 \pm 0.2$ & $1.0 \pm 0.4$ & $1.2 \pm 0.6^{\mathrm{b}}$ & $0.8 \pm 0.3^{f}$ & $<0.001$ \\
\hline Total cholesterol $[\mathrm{mmol} / \mathrm{L}]$ & $4.5 \pm 0.95$ & $4.7 \pm 0.97$ & $4.7 \pm 1.0$ & $4.8 \pm 0.65$ & 0.294 \\
\hline Triglyceride $[\mathrm{mmol} / \mathrm{L}]$ & $1.8 \pm 0.8$ & $2.0 \pm 0.8$ & $1.5 \pm 0.6$ & $1.7 \pm 0.4$ & 0.108 \\
\hline Creatinine $[\mu \mathrm{mol} / \mathrm{L}]$ & $703 \pm 170$ & $733 \pm 125$ & $80 \pm 18^{b, d}$ & $79 \pm 15^{\mathrm{c}, \mathrm{e}}$ & $<0.001$ \\
\hline Blood urea nitrogen [mmol/L] & $27 \pm 6$ & $27 \pm 5$ & $5.5 \pm 1.7^{\mathrm{b}, \mathrm{d}}$ & $5.3 \pm 1.1^{c, e}$ & $<0.001$ \\
\hline Total calcium $[\mathrm{mmol} / \mathrm{L}]$ & $2.1 \pm 0.4$ & $2.2 \pm 0.2$ & $2.2 \pm 0.1$ & $2.2 \pm 0.2$ & 0.698 \\
\hline Iron $[\mu \mathrm{mol} / \mathrm{L}]$ & $18 \pm 9$ & $13 \pm 6^{1}$ & $18 \pm 3$ & $20 \pm 6^{5}$ & 0.005 \\
\hline Fasting glucose $[\mathrm{mmol} / \mathrm{L}]$ & $7.2 \pm 4.0$ & $6.2 \pm 3.2$ & $5.5 \pm 0.6$ & $5.7 \pm 1.9$ & 0.062 \\
\hline Albumin $[\mathrm{g} / \mathrm{L}]$ & $40 \pm 4$ & $40 \pm 3$ & $38 \pm 2$ & $37 \pm 4^{3}$ & 0.003 \\
\hline Total proteins $[\mathrm{g} / \mathrm{L}]$ & $65 \pm 5$ & $64 \pm 6$ & $66 \pm 2$ & $66 \pm 3$ & 0.213 \\
\hline Aspartate aminotransferase $[\mathrm{U} / \mathrm{L}]$ & $22 \pm 18$ & $15 \pm 5$ & $18 \pm 6$ & $18 \pm 5$ & 0.169 \\
\hline Alanine aminotransferase $[\mathrm{U} / \mathrm{L}]$ & $26 \pm 23$ & $16 \pm 9$ & $19 \pm 8$ & $20 \pm 8$ & 0.078 \\
\hline Red blood cells $\left[\times 10^{6} / \mathrm{uL}\right]$ & $3.4 \pm 0.7$ & $3.6 \pm 1.2$ & $4.3 \pm 0.6^{\mathrm{b}, 4}$ & $4.7 \pm 0.5^{\mathrm{c}, \mathrm{e}}$ & $<0.001$ \\
\hline White blood cells $\left[\times 10^{3} / \mathrm{uL}\right]$ & $7.6 \pm 3.7$ & $7.0 \pm 1.3$ & $7.5 \pm 1.3$ & $7.8 \pm 1.2$ & 0.755 \\
\hline Hematocrit [\%] & $33 \pm 5.5$ & $32 \pm 5.4$ & $38 \pm 4.2^{2,4}$ & $40 \pm 7.3^{\mathrm{c}, \mathrm{e}}$ & $<0.001$ \\
\hline Hemoglobin $[\mathrm{g} / \mathrm{dL}]$ & $10 \pm 1.7$ & $10 \pm 1.5$ & $13 \pm 1.6^{\mathrm{b}, \mathrm{d}}$ & $14 \pm 0.8^{c, e}$ & $<0.001$ \\
\hline
\end{tabular}

(1): $p<0.05$ gr. 1 vs. gr. 2 ; (2): $p<0.05$ gr. 1 vs. gr. 3 ; (3): $p<0.05$ gr. 1 vs. gr. 4 ; (4): $p<0.05$ gr. 2 vs. gr. 3 ; (5): $p<0.05$ gr. 2 vs. gr. 4 ; (6): $p<0.05$ gr. 3 vs. gr. 4

(a): $p<0.001$ gr. 1 vs. gr. 2 ; (b): $p<0.001$ gr. 1 vs. gr. 3 ; (c): $p<0.001$ gr. 1 vs. gr. 4 ; (d) $p<0.001$ gr. 2 vs. gr. 3 ; (e): $p<0.001$ gr. 2 vs. gr. 4 ; (f): $p<0.001$ gr. 3 vs. gr. 4

Gr. 1 - dialysis patients with LV hypertrophy; Gr. 2 - dialysis patients without LV hypertrophy; Gr. 3 - non-dialysis with LV hypertrophy; Gr. 4 - non-dialysis without LV hypertrophy

LV — left ventricular; SBP — systolic blood pressure; DBP — diastolic blood pressure; ACE — angiotensin converting enzyme; HDL — high-density lipoprotein

Dialysis patients with $\mathrm{LVH}$ versus non-dialysis patients with LVH (Tables 3. 4)

Dialysis patients with LVH, had higher adiponectin (Fig. 1), lower HDL-C, lower hemoglobin and RBC, shorter LVET ( $p<0.001$, for all), lower weight/hip ratio, lower BMI and BSA, lower hematocrit, lower baseline heart rate and broader QRS complex ( $p<0.05$ for all), compared to non-dialysis LVH patients. All other clinical, biochemical and echocardiographic indices did not differ between the two groups.
Relationship of adiponectin with clinical, biochemical and cardiac function indices In dialysis patients versus controls (Table 5)

In all study patients, adiponectin had strong correlation with LVMI $(\mathrm{p}<0.001)$ (Fig. 2). In dialysis patients, adiponectin had a strong correlation with anthropometric parameters (waist measures, BMI and BSA, p < 0.001, for all) (Figs. 3, 4), good correlation with LVMI ( $\mathrm{p}=0.005)$ (Fig. 5), but weak correlation with waist/hips ratio $(\mathrm{p}=0.037)$ and with total cholesterol level $(\mathrm{p}=0.013)$. On the 
Table 4. Echocardiographic data of study subjects.

\begin{tabular}{|c|c|c|c|c|c|}
\hline Variable & $\begin{array}{l}\text { Group } 1 \\
(n=65)\end{array}$ & $\begin{array}{l}\text { Group } 2 \\
(n=24)\end{array}$ & $\begin{array}{l}\text { Group } 3 \\
(n=25)\end{array}$ & $\begin{array}{l}\text { Group } 4 \\
(n=30)\end{array}$ & $\mathbf{P}$ \\
\hline \multicolumn{6}{|l|}{ LV dimension and mass } \\
\hline LVM [g] & $245 \pm 66$ & $190 \pm 45^{1}$ & $237 \pm 100$ & $196 \pm 39^{3}$ & $<0.001$ \\
\hline LVMI $\left[\mathrm{g} / \mathrm{m}^{2.7}\right]$ & $78 \pm 27$ & $41 \pm 5^{a}$ & $59 \pm 10^{\mathrm{b}, 4}$ & $38 \pm 7^{c, f}$ & $<0.001$ \\
\hline Interventricular septum [cm] & $1.3 \pm 0.25$ & $1.1 \pm 0.14$ & $1.2 \pm 0.17$ & $1.1 \pm 0.14^{3}$ & $<0.001$ \\
\hline LV posterior wall [cm] & $1.1 \pm 0.17$ & $1.0 \pm 0.13$ & $1.0 \pm 0.13$ & $1.0 \pm 0.14$ & 0.039 \\
\hline End diastolic volume [mL] & $125 \pm 37$ & $105 \pm 26^{1}$ & $115 \pm 21$ & $112 \pm 23$ & 0.032 \\
\hline End systolic volume [mL] & $50 \pm 24$ & $44 \pm 21$ & $43 \pm 144$ & $35 \pm 13^{3}$ & 0.016 \\
\hline \multicolumn{6}{|l|}{ LV systolic function } \\
\hline LV ejection fraction [\%] & $61 \pm 8$ & $59 \pm 12^{1}$ & $62 \pm 7$ & $66 \pm 8^{3,5}$ & 0.012 \\
\hline LV shortening fraction [\%] & $33 \pm 6$ & $31 \pm 8$ & $33 \pm 5$ & $35 \pm 5$ & 0.099 \\
\hline Stroke volume $[\mathrm{mL}]$ & $75 \pm 19$ & $61 \pm 18^{1}$ & $68 \pm 22$ & $74 \pm 17$ & 0.010 \\
\hline Filling time [ms] & $391 \pm 95$ & $375 \pm 74$ & $434 \pm 117$ & $397 \pm 80$ & 0.144 \\
\hline Ejection time [ms] & $278 \pm 43$ & $282 \pm 40$ & $324 \pm 50^{\mathrm{b}, 4}$ & $308 \pm 33^{3}$ & $<0.001$ \\
\hline Tei index & $0.39 \pm 0.3$ & $0.30 \pm 0.2$ & $0.34 \pm 0.2$ & $0.40 \pm 0.2$ & 0.396 \\
\hline $\mathrm{t}-\mathrm{IVT}[\mathrm{ms}]$ & $7.8 \pm 3.8$ & $6.8 \pm 3.3$ & $6.6 \pm 4$ & $8.7 \pm 4.5$ & 0.162 \\
\hline Lateral s' $[\mathrm{cm}]$ & $6.9 \pm 1.9$ & $6.7 \pm 1.6$ & $6.5 \pm 1.7$ & $7.5 \pm 1.9$ & 0.189 \\
\hline Septal s' $[\mathrm{cm} / \mathrm{s}]$ & $5.5 \pm 1.4$ & $6.5 \pm 1.5$ & $5.3 \pm 1.0$ & $6.8 \pm 1.5$ & $<0.001$ \\
\hline Septal MAPSE [cm] & $1.3 \pm 0.3$ & $1.4 \pm 0.3$ & $1.4 \pm 0.3$ & $1.5 \pm 0.3^{3}$ & 0.014 \\
\hline Lateral MAPSE [cm] & $1.5 \pm 0.3$ & $1.6 \pm 0.3$ & $1.6 \pm 0.3$ & $1.6 \pm 0.2$ & 0.199 \\
\hline \multicolumn{6}{|l|}{ LV diastolic function } \\
\hline IVRT [ms] & $128 \pm 32$ & $127 \pm 28$ & $100 \pm 29^{2,4}$ & $102 \pm 27^{c, 5}$ & $<0.001$ \\
\hline E wave $[\mathrm{cm} / \mathrm{s}]$ & $57 \pm 20$ & $53 \pm 12$ & $56 \pm 13$ & $58 \pm 15^{3}$ & 0.734 \\
\hline A wave $[\mathrm{cm} / \mathrm{s}]$ & $73 \pm 19$ & $70 \pm 14$ & $68 \pm 17$ & $61 \pm 15$ & 0.021 \\
\hline$E / A$ & $0.9 \pm 0.7$ & $0.8 \pm 0.3$ & $0.8 \pm 0.3$ & $1.0 \pm 0.7$ & 0.729 \\
\hline EDT [ms] & $185 \pm 53$ & $163 \pm 38$ & $176 \pm 46$ & $171 \pm 34$ & 0.241 \\
\hline Lateral $\mathrm{e}^{\prime}[\mathrm{cm} / \mathrm{s}]$ & $7.7 \pm 3.2$ & $8.2 \pm 3.7$ & $7.3 \pm 2.2$ & $8.7 \pm 3.3$ & 0.323 \\
\hline Lateral a' $[\mathrm{cm} / \mathrm{s}]$ & $9.1 \pm 2.7$ & $10 \pm 3.2$ & $9.1 \pm 2.4$ & $9.2 \pm 2.4$ & 0.458 \\
\hline Lateral s' $[\mathrm{cm} / \mathrm{s}]$ & $6.9 \pm 1.9$ & $6.7 \pm 1.6$ & $6.5 \pm 1.7$ & $7.5 \pm 1.9$ & 0.189 \\
\hline \multicolumn{6}{|l|}{ Septal e' $[\mathrm{cm} / \mathrm{s}]$} \\
\hline Septal a' $[\mathrm{cm} / \mathrm{s}]$ & $8 \pm 2.4$ & $9 \pm 2.6$ & $8 \pm 1.6$ & $8 \pm 1.3$ & 0.182 \\
\hline Septal s' $[\mathrm{cm} / \mathrm{s}]$ & $6 \pm 1.4$ & $6 \pm 1.5$ & $5 \pm 1.0^{4}$ & $7 \pm 1.5^{\mathrm{c}, \mathrm{f}}$ & $<0.001$ \\
\hline \multicolumn{6}{|l|}{ LA dimension and function } \\
\hline LA diameter $[\mathrm{cm}]$ & $3.9 \pm 0.5$ & $3.8 \pm 0.5$ & $3.7 \pm 0.3$ & $3.7 \pm 0.3$ & 0.383 \\
\hline LA maximal volume $[\mathrm{mL}]$ & $52.5 \pm 16$ & $61 \pm 28$ & $56 \pm 23$ & $53 \pm 20$ & 0.558 \\
\hline LA minimal volume $[\mathrm{mL}]$ & $19 \pm 9$ & $26 \pm 17$ & $23 \pm 13$ & $24 \pm 15$ & 0.353 \\
\hline LA emptying fraction [\%] & $65 \pm 10$ & $62 \pm 11$ & $61 \pm 9$ & $56 \pm 13$ & 0.080 \\
\hline \multicolumn{6}{|l|}{ Aortic measurement } \\
\hline Aorta $[\mathrm{cm}]$ & $3.6 \pm 0.4$ & $3.5 \pm 0.4$ & $3.4 \pm 0.3$ & $3.4 \pm 0.3$ & 0.704 \\
\hline
\end{tabular}

(1): $p<0.05$ gr. 1 vs. gr. $2 ;(2): p<0.05$ gr. 1 vs. gr. 3; (3): $p<0.05$ gr. 1 vs. gr. 4; (4): p < 0.05 gr. 2 vs. gr. 3; (5): $p<0.05$ gr. 2 vs. gr. 4 ; (6): $p<0.05$ gr. 3 vs. gr. 4

(a): $p<0.001$ gr. 1 vs. gr. 2 ; (b): $p<0.001$ gr. 1 vs. gr. 3 ; (c): $p<0.001$ gr. 1 vs. gr. 4 ; (d): $p<0.001$ gr. 2 vs. gr. 3 ; (e): $p<0.001$ gr. 2 vs. gr. 4 ; (f): $\mathrm{p}<0.001$ gr. 3 vs. gr. 4

Gr. 1 - hemodialysis with LV hypertrophy; Gr. 2 - emodialysis without LV hypertrophy; Gr. 3 - non hemodialysis with LV hypertrophy; Group 4 - non hemodialysis without LV hypertrophy

LV - left ventricular; LVM - left ventricular mass; LVMI — left ventricular mass index; LA — left atrium; RV — right ventricle; MAPSE — mitral annular plane systolic excursion; IVRT — isovolumic relaxation time; $t$-IVT — total isovolumic time; EDT — E wave deceleration time; A - atrial diastolic velocity; a' - late diastolic myocardial velocity; E - early mitral inflow velocity; $\mathrm{e}^{\prime}$ - early diastolic myocardial velocity; $\mathrm{s}^{\prime}$ - systolic myocardial velocity 


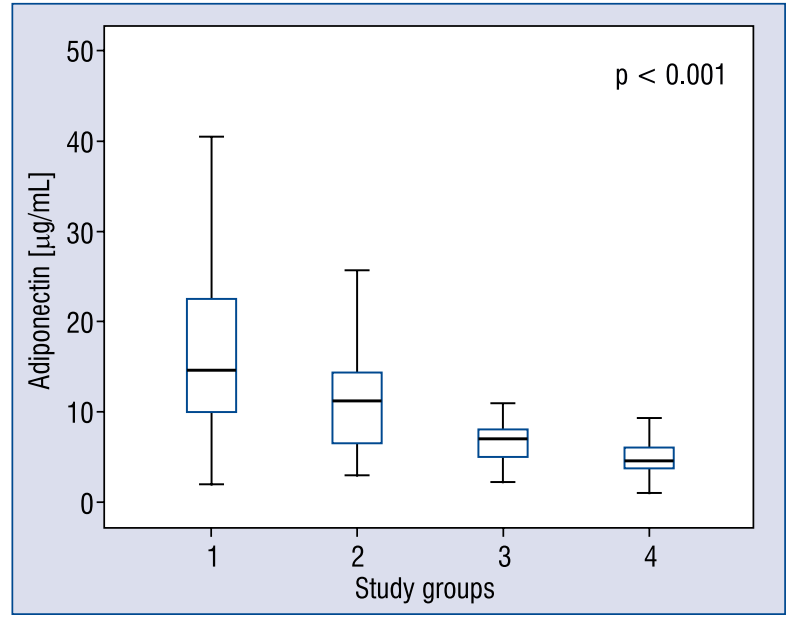

Figure 1. Adiponectin level in study groups of patients; 1 - Group 1 (dialysis patients with LVH; 2 - Group 2 (dialysis patients without LVH); 3 - Group 3 (non-dialysis patients with $L V H$ ); 4 - Group 4 (non-dialysis patients without LVH); LVH — left ventricular hypertherapy.

other hand, in controls, adiponectin only modestly correlated with age $(\mathrm{p}=0.024)$, hemoglobin $(\mathrm{p}=$ $0.005)$, HDL-C ( $=0.019)$, and LVMI $(\mathrm{p}=0.043)$ (Fig. 5), but no relationship was noted with the other clinical, biochemical and echocardiographic parameters.

\section{Discussion}

The findings of this study can be summarized as follows: dialysis patients had lower BSA and BMI compared to age and gender matched controls but did have a larger LV cavity with thicker walls, higher LVMI and broader QRS duration. In addition, they were more anemic and had higher adiponectin levels compared to controls. Finally, while in dialysis patients adiponectin strongly correlated with BMI, it only modestly correlated with LVMI and volumes. The only relevant relationship in controls was with LVMI, although to a lesser strength than with dialysis patients.

Data interpretation. As expected, dialysis patients had lower body weight and BMI consistent with an element of cachexia, which is well known in patients with chronic kidney disease [39]. The profound degree of LVH resulting in higher mass index, again is consistent with the commonly found hypertension in this condition [40]. Such changes in LV structure resulted in significant functional disturbances in the form of reduced subendo-

Table 5. Correlation of adiponectin with clinical, biochemical and echocardiographic variables in study patients.

\begin{tabular}{|c|c|c|c|c|c|c|}
\hline \multirow[t]{2}{*}{ Variable } & \multicolumn{2}{|c|}{$\begin{array}{l}\text { All study patients } \\
\quad(\mathrm{n}=145)\end{array}$} & \multicolumn{2}{|c|}{$\begin{array}{l}\text { Controls } \\
(\mathrm{n}=55)\end{array}$} & \multicolumn{2}{|c|}{$\begin{array}{l}\text { Dialysis patients } \\
\quad(\mathrm{n}=\mathbf{8 9})\end{array}$} \\
\hline & $\mathbf{r}$ & $\mathbf{p}$ & $\mathbf{r}$ & $\mathbf{p}$ & $\mathbf{r}$ & $\mathbf{p}$ \\
\hline Age & 0.008 & 0.922 & 0.304 & 0.024 & 0.056 & 0.599 \\
\hline SBP & 0.184 & 0.027 & 0.122 & 0.373 & 0.042 & 0.697 \\
\hline DBP & -0.099 & 0.238 & 0.075 & 0.586 & -0.001 & 0.993 \\
\hline C-reactive protein & 0.068 & 0.420 & 0.211 & 0.121 & 0.112 & 0.298 \\
\hline Cholesterol & -0.252 & 0.002 & 0.052 & 0.707 & -0.292 & 0.013 \\
\hline HDL cholesterol & 0.051 & 0.545 & 0.315 & 0.019 & 0.195 & 0.067 \\
\hline Hemoglobin & -0.427 & $<0.001$ & 0.371 & 0.005 & -0.020 & 0.853 \\
\hline Urea & 0.499 & $<0.001$ & -0.153 & 0.266 & -0.037 & 0.730 \\
\hline Creatinine & 0.494 & $<0.001$ & -0.084 & 0.544 & -0.066 & 0.541 \\
\hline Waist & -0.461 & $<0.001$ & -0.141 & 0.304 & -0.427 & $<0.001$ \\
\hline Waist/hips ratio & -0.283 & 0.001 & -0.184 & 0.178 & -0.222 & 0.037 \\
\hline Body mass index & -0.468 & $<0.001$ & -0.093 & 0.498 & -0.403 & $<0.001$ \\
\hline Body surface area & -0.522 & $<0.001$ & -0.096 & 0.487 & -0.480 & $<0.001$ \\
\hline Left atrium & 0.065 & 0.437 & -0.075 & 0.585 & 0.001 & 0.996 \\
\hline Ejection fraction & -0.107 & 0.201 & -0.162 & 0.237 & 0.044 & 0.684 \\
\hline Left ventricular mass & 0.076 & 0.369 & 0.178 & 0.197 & -0.013 & 0.904 \\
\hline LVMI & 0.440 & $<0.001$ & 0.277 & 0.043 & 0.296 & 0.005 \\
\hline
\end{tabular}

SBP — systolic blood pressure; DBP — diastolic blood pressure; HDL — high-density lipoprotein; LVMI — left ventricular mass index 


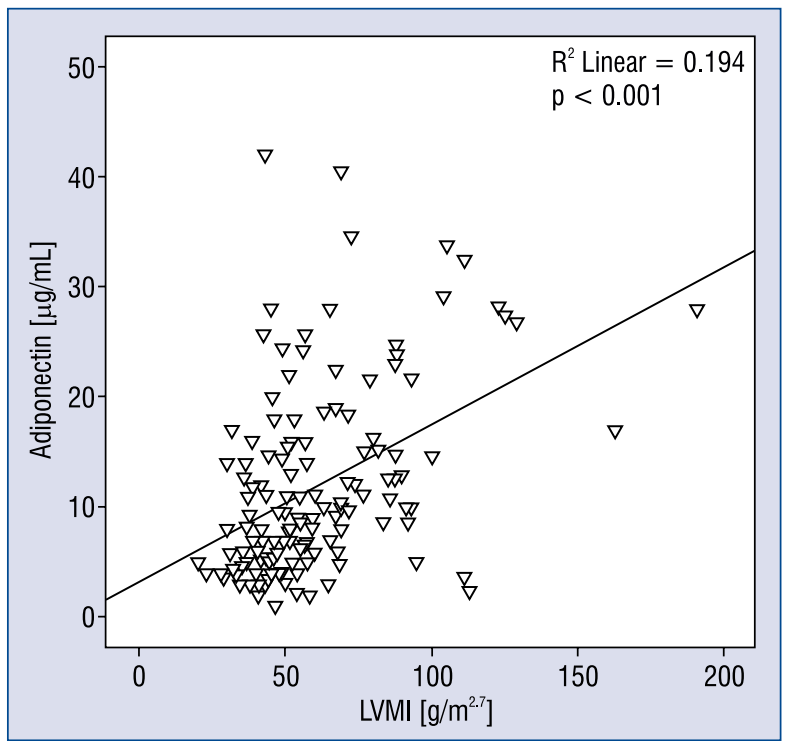

Figure 2. Correlation of adiponectin with left ventricular mass index (LVMI) in all study patients.

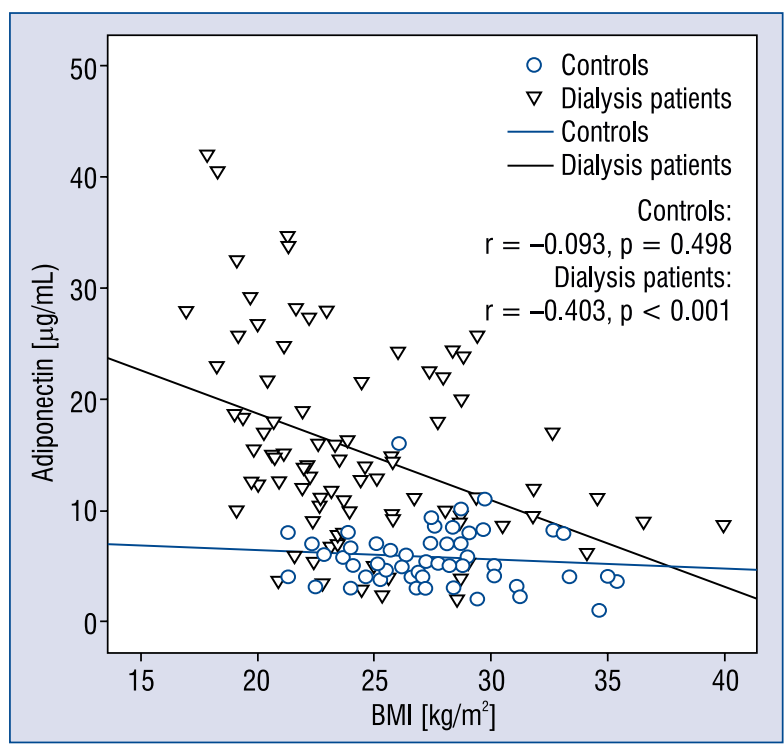

Figure 3. Correlation of adiponectin with body mass index (BMI) in dialysis patients and controls.

cardial function reflected on the MAPSE and its myocardial velocities, enlargement of LV volume, lower ejection fraction and left atrial enlargement, typical to what is commonly seen in hypertensive LV disease without kidney failure [41]. Furthermore, the dialysis patients had some degree of dyssynchrony in the form of broader QRS, along with shorter ejection and filling times, caused by prolonged isovolumic times. The enlargement

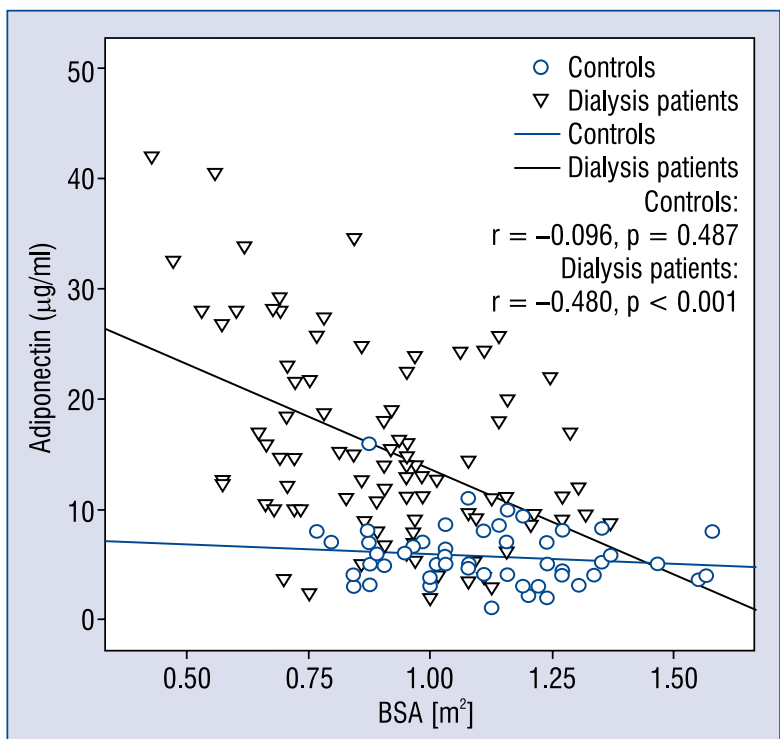

Figure 4. Correlation of adiponectin with body surface area (BSA) in dialysis patients and controls.

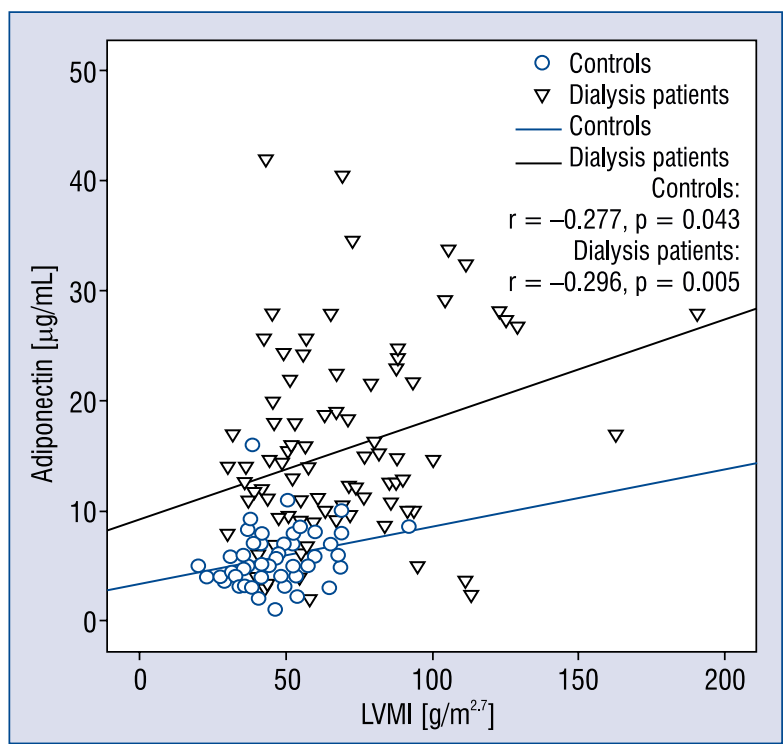

Figure 5. Correlation of adiponectin with left ventricular mass index (LVMI) in dialysis patients and controls.

of LV volume could be interpreted on the basis of volume overload because of dialysis. Despite all these changes in LV structure and function adponectine did not seem to be related to any of them, except weakly with LVMI. Only few studies exist in the literature, that assessed a relationship between LVMI and adiponectin in dialysis patients. These studies include a small number of patients and their direct comparison with the present re- 
sults has limitations. Komaba et al. [4] included only diabetic patients with dialysis, and Amira et al. [42] found good correlation, whereas Ayerden Ebinç et al. [23] was the only study whose results were in line with ours, having found a weak correlation between adiponectin and LVMI in dialysis patients. The strongest relationship found herein was between adiponectin and BMI, which is a reliable measure of body fat. These results support the close relationship of adiponectin and its site of secretion, i.e. the adipose tissue, rather than with cardiac structure and function.

\section{Limitations of the study}

As suggested above, the potential effect of volume overload during dialysis on the LV causing cavity enlargement, this might also have affected left atrial size and LV filling velocities and timing, hence the lack of a relationship with adiponectine. Right heart structure and function in this analysis was not included which might have shed some light on potential relationships, although they are also likely to be affected by loading conditions.

\section{Conclusions}

Although adiponectin is an established cardioprotective marker, it does not seem to be related to cardiac structure and function parameters but only strongly to BMI suggesting an ongoing catabolic process associating dialysis.

\section{Conflict of interest: None declared}

\section{References}

1. Mitsuhashi H, Yatsuya H, Tamakoshi K, et al. Adiponectin level and left ventricular hypertrophy in Japanese men. Hypertens Dallas Tex 1979. 2007; 49(6): 1448-1454, doi: 10.1161/HYPERTENSIONAHA.106.079509, indexed in Pubmed: 17420337.

2. Wiecek A, Adamczak M, Chudek J. Adiponectin--an adipokine with unique metabolic properties. Nephrol Dial Transplant. 2007; 22(4): 981-988, doi: 10.1093/ndt/gfl814, indexed in Pubmed: 17234664.

3. Ouchi N, Kihara S, Arita Y, et al. Novel modulator for endothelial adhesion molecules: adipocyte-derived plasma protein adiponectin. Circulation. 1999; 100(25): 2473-2476, indexed in Pubmed: 10604883.

4. Komaba H, Igaki N, Goto S, et al. Adiponectin is associated with brain natriuretic peptide and left ventricular hypertrophy in hemodialysis patients with type 2 diabetes mellitus. Nephron Clin Pract. 2007; 107(3): c103-c108, doi: 10.1159/000108651, indexed in Pubmed: 17890872.

5. Scherer PE, Williams S, Fogliano M, et al. A novel serum protein similar to C1q, produced exclusively in adipocytes. J Biol Chem. 1995; 270(45): 26746-26749, indexed in Pubmed: 7592907.
6. Goldstein BJ, Scalia R. Adiponectin: A novel adipokine linking adipocytes and vascular function. J Clin Endocrinol Metab. 2004; 89(6): 2563-2568, doi: 10.1210/jc.2004-0518, indexed in Pubmed: 15181024.

7. Ebinç H, Ebinç FA, Ozkurt ZN, et al. Impact of adiponectin on left ventricular mass index in non-complicated obese subjects. Endocr J. 2008; 55(3): 523-528, indexed in Pubmed: 18469485.

8. Weyer C, Funahashi T, Tanaka S, et al. Hypoadiponectinemia in obesity and type 2 diabetes: close association with insulin resistance and hyperinsulinemia. J Clin Endocrinol Metab. 2001; 86(5): 1930-1935, doi: 10.1210/jcem.86.5.7463, indexed in Pubmed: 11344187 .

9. Matsubara M, Maruoka S, Katayose S. Decreased plasma adiponectin concentrations in women with dyslipidemia. J Clin Endocrinol Metab. 2002; 87(6): 2764-2769, doi: 10.1210/ jcem.87.6.8550, indexed in Pubmed: 12050247.

10. Hotta K, Funahashi T, Arita Y, et al. Plasma concentrations of a novel, adipose-specific protein, adiponectin, in type 2 diabetic patients. Arterioscler Thromb Vasc Biol. 2000; 20(6): 1595-1599, indexed in Pubmed: 10845877.

11. Della Mea P, Lupia M, Bandolin V, et al. Adiponectin, insulin resistance, and left ventricular structure in dipper and nondipper essential hypertensive patients. Am J Hypertens. 2005; 18(1): 30-35, doi: 10.1016/j.amjhyper.2004.08.029, indexed in Pubmed: 15691614.

12. Foley RN. Clinical epidemiology of cardiac disease in dialysis patients: left ventricular hypertrophy, ischemic heart disease, and cardiac failure. Semin Dial. 2003; 16(2): 111-117, indexed in Pubmed: 12641874.

13. Pischon T, Girman CJ, Hotamisligil GS, et al. Plasma adiponectin levels and risk of myocardial infarction in men. JAMA. 2004; 291(14): 1730-1737, doi: 10.1001/jama.291.14.1730, indexed in Pubmed: 15082700.

14. Shibata R, Ouchi N, Ito M, et al. Adiponectin-mediated modulation of hypertrophic signals in the heart. Nat Med. 2004; 10(12): 1384-1389, doi: 10.1038/nm1137, indexed in Pubmed: 15558058.

15. Levy D, Garrison RJ, Savage DD, et al. Prognostic implications of echocardiographically determined left ventricular mass in the Framingham Heart Study. N Engl J Med. 1990; 322(22): 1561-1566, doi: 10.1056/NEJM199005313222203, indexed in Pubmed: 2139921.

16. Verdecchia P, Schillaci G, Borgioni C, et al. Prognostic significance of serial changes in left ventricular mass in essential hypertension. Circulation. 1998; 97(1): 48-54, indexed in Pubmed: 9443431.

17. Stack AG, Saran R. Clinical correlates and mortality impact of left ventricular hypertrophy among new ESRD patients in the United States. Am J Kidney Dis. 2002; 40(6): 1202-1210, doi: 10.1053/ajkd.2002.36881, indexed in Pubmed: 12460039.

18. Silberberg JS, Barre PE, Prichard SS, et al. Impact of left ventricular hypertrophy on survival in end-stage renal disease. Kidney Int. 1989; 36(2): 286-290, indexed in Pubmed: 2528654.

19. Mallamaci F, Zoccali C, Cuzzola F, et al. Adiponectin in essential hypertension. J Nephrol. 2002; 15(5): 507-511, indexed in Pubmed: 12455716.

20. Kazumi T, Kawaguchi A, Sakai K, et al. Young men with highnormal blood pressure have lower serum adiponectin, smaller LDL size, and higher elevated heart rate than those with optimal blood pressure. Diabetes Care. 2002; 25(6): 971-976, indexed in Pubmed: 12032101. 
21. Adamczak M, Wiecek A, Funahashi T, et al. Decreased plasma adiponectin concentration in patients with essential hypertension. Am J Hypertens. 2003; 16(1): 72-75, indexed in Pubmed: 12517687.

22. Furuhashi M, Ura N, Higashiura K, et al. Blockade of the renin-angiotensin system increases adiponectin concentrations in patients with essential hypertension. Hypertens Dallas Tex. 2003; 42(1): 76-81, doi: 10.1161/01.HYP.0000078490.59735.6E, indexed in Pubmed: 12796280.

23. Ayerden Ebinç H, Derici U, et al. The relationship between adiponectin levels and proinflammatory cytokines and left ventricular mass in dialysis patients. J Nephrol. 2009; 22(2): 216-223.

24. Becker B, Kronenberg F, Kielstein JT, et al. MMKD Study Group. Renal insulin resistance syndrome, adiponectin and cardiovascular events in patients with kidney disease: the mild and moderate kidney disease study. J Am Soc Nephrol. 2005; 16(4): 1091-1098, doi: 10.1681/ASN.2004090742, indexed in Pubmed: 15743998.

25. Frystyk J, Tarnow L, Hansen TK, et al. Increased serum adiponectin levels in type 1 diabetic patients with microvascular complications. Diabetologia. 2005; 48(9): 1911-1918, doi: 10.1007/s00125-005-1850-z, indexed in Pubmed: 16078018.

26. Komaba H, Igaki N, Goto S, et al. Increased serum high-molecular-weight complex of adiponectin in type 2 diabetic patients with impaired renal function. Am J Nephrol. 2006; 26(5): 476-482, doi: 10.1159/000096870, indexed in Pubmed: 17095862.

27. Zoccali C, Mallamaci F, Tripepi G, et al. Adiponectin, metabolic risk factors, and cardiovascular events among patients with endstage renal disease. J Am Soc Nephrol. 2002; 13(1): 134-141, indexed in Pubmed: 11752030.

28. Tanita T, Miyakoshi H, Nakano Y. Performance of ELISA for specific measurement of high-molecular-weight (HMW) adiponectin. J Immunol Methods. 2008; 333(1-2): 139-146, doi: 10.1016/j. jim.2008.01.013, indexed in Pubmed: 18304569.

29. Devereux RB, Alonso DR, Lutas EM, et al. Echocardiographic assessment of left ventricular hypertrophy: comparison to necropsy findings. Am J Cardiol. 1986; 57(6): 450-458, indexed in Pubmed: 2936235.

30. Devereux RB, Reichek N. Echocardiographic determination of left ventricular mass in man. Anatomic validation of the method. Circulation. 1977; 55(4): 613-618, indexed in Pubmed: 138494.

31. de Simone G, Daniels SR, Devereux RB, et al. Left ventricular mass and body size in normotensive children and adults: assessment of allometric relations and impact of overweight. J Am Coll Cardiol. 1992; 20(5): 1251-1260, indexed in Pubmed: 1401629.

32. Gosse P, Jullien V, Jarnier P, et al. Echocardiographic definition of left ventricular hypertrophy in the hypertensive: which method of indexation of left ventricular mass? J Hum Hypertens. 1999; 13(8): 505-509, indexed in Pubmed: 10455470.
33. Bajraktari G, Berbatovci-Ukimeraj M, Hajdari A, et al. Predictors of increased left ventricular filling pressure in dialysis patients with preserved left ventricular ejection fraction. Croat Med J. 2009; 50(6): 543-549, indexed in Pubmed: 20017222.

34. Özkan B, Açar G, Alıcı G, et al. Decreased plasma adiponectin is associated with impaired left ventricular longitudinal systolic function in hypertensive patients: a two-dimensional speckle tracking study. Clin Exp Hypertens. 2014; 36(1): 46-51, doi: 10.3109/10641963.2013.783053, indexed in Pubmed: 23772851.

35. Duncan AM, Francis DP, Henein MY, et al. Importance of left ventricular activation in determining myocardial performance (Tei) index: comparison with total isovolumic time. Int J Cardiol. 2004; 95(2-3): 211-217, doi: 10.1016/j.ijcard.2003.07.007, indexed in Pubmed: 15193822.

36. Tei C, Ling LH, Hodge DO, et al. New index of combined systolic and diastolic myocardial performance: a simple and reproducible measure of cardiac function--a study in normals and dilated cardiomyopathy. J Cardiol. 1995; 26(6): 357-366, indexed in Pubmed: 8558414.

37. Galderisi M, Henein MY, D’hooge J, et al. Recommendations of the European Association of Echocardiography: how to use echoDoppler in clinical trials: different modalities for different purposes. Eur J Echocardiogr. 2011; 12(5): 339-353, doi: 10.1093/ ejechocard/jer051, indexed in Pubmed: 21555455.

38. Lang RM, Bierig M, Devereux RB, et al. Recommendations for chamber quantification: a report from the American Society of Echocardiography's Guidelines and Standards Committee and the Chamber Quantification Writing Group, developed in conjunction with the European Association of Echocardiography, a branch of the European Society of Cardiology. J Am Soc Echocardiogr. 2005; 18(12): 1440-1463, doi: 10.1016/j.echo.2005.10.005, indexed in Pubmed: 16376782.

39. Yoshida T, Delafontaine P. Mechanisms of cachexia in chronic disease states. Am J Med Sci. 2015; 350(4): 250-256, doi: 10.1097/ MAJ.0000000000000511, indexed in Pubmed: 26083652.

40. Paoletti E, De Nicola L, Gabbai FB, et al. Associations of left ventricular hypertrophy and geometry with adverse outcomes in patients with CKD and hypertension. Clin J Am Soc Nephrol. 2016; 11(2): 271-279, doi: 10.2215/CJN.06980615, indexed in Pubmed: 26668021.

41. Koulouris SN, Kostopoulos KG, Triantafyllou KA, et al. Impaired systolic dysfunction of left ventricular longitudinal fibers: a sign of early hypertensive cardiomyopathy. Clin Cardiol. 2005; 28(6): 282-286, indexed in Pubmed: 16028462.

42. Amira OC, Naicker S, Manga P, et al. Adiponectin and atherosclerosis risk factors in African hemodialysis patients: a population at low risk for atherosclerotic cardiovascular disease. Hemodial Int Int Symp Home Hemodial. 2012; 16(1): 59-68, doi: 10.1111/j.15424758.2011.00622.x, indexed in Pubmed: 22099423. 